\title{
Comment j'utilise les médias sociaux dans mes cours à l’université
}

\section{Cómo utilizo la web social en mis clases de la Universidad How I use the social web in my university courses}

\author{
Pierre Lévy \\ Université d'Ottawa. Otawa. Canadá \\ plevy@uOttawa.ca
}

\begin{abstract}
L'article témoigne de plusieurs années d'expérience d'enseignement à l'université en utilisant les médias sociaux (Facebook, Twitter, blogs). Les étudiants sont appelés à prendre leurs notes en temps réel sur Twitter afin de constituer un cahier de notes collectif repéré par le hashtag du cours. Ils sont également entraînés à pratiquer la curation: choisir, catégoriser et commenter en ligne des documents pertinents. Ils doivent ensuite relire cette mémoire collective, en extraire les meilleurs moments et réfleéchir à leur apprentissage
\end{abstract}

\section{Mots clés}

Université, pédagogie, médias sociaux, intelligence collective, curation, Twitter, blogs

\section{Resumen}

El artículo muestra, en clave de «historia personal como investigación educativa» (Personal History as Educational Research), varios años de experiencia docente en la universidad utilizando las redes sociales (Facebook, Twitter, blogs). Se les pide a los estudiantes que tomen sus notas en tiempo real, en Twitter, para crear un cuaderno colectivo marcado con el hashtag del curso. También están entrenados para practicar la curaduría: elegir, categorizar y comentar documentos relevantes en línea. Luego deben volver a leer esta memoria colectiva, extraer los mejores momentos y reflexionar sobre su aprendizaje.

\section{Keywords}

Educación universitaria, pedagogía, web social, inteligencia colectiva, curacion, Twitter, blog.

\footnotetext{
Abstract

This paper reports several years of teaching experience at the university using social media (Facebook, Twitter, blogs). Students are asked to take their notes in real time on Twitter in order to create a collective notebook signaled by the hashtag of the course. They are also trained to curation: choosing, categorizing and commenting online relevant documents. They must then review their collective memory, extract the best moments and reflect on their learning.
}

\section{Keywords}

University, pedagogy, social media, collective intelligence, curation, Twitter, blog 
Cet article n'a d'autre but que de raconter mon expérience d'enseignement avec les médias sociaux dans mes cours de communication à l'Université d'Ottawa. Je ne prétends nullement servir de modèle. Si j'avais cependant deux conseils à donner, je recommanderais d'abord aux enseignants de toujours penser à l'utilisation des médias sociaux dans une perspective pédagogique, en les intégrant dès l'origine dans le design de leur cours et dans l'évaluation des étudiants. Mon second conseil est de ne jamais appliquer une méthode toute faite. Ils devront plutôt acquérir par l'expérience une maîtrise de l'apprentissage collaboratif dans les médias sociaux puis amener les étudiants le plus près possible de leur propre niveau. La méthode changera donc avec le degré d'apprentissage de l'enseignant et devra tenir compte du savoir-faire des étudiants, du contexte disciplinaire, social, etc. Le chemin d'apprentissage personnel de l'enseignant joue un rôle essentiel dans la forme et la qualité de son enseignement.

Dans les cours que je donne à l'Université d'Ottawa, je demande à mes étudiants de participer à un groupe Facebook fermé, de s'enregistrer sur Twitter, d'ouvrir un blog s'ils n'en n'ont pas déjà un et d'utiliser une plateforme de curation collaborative de données comme Scoop.it, Diigo ou Pocket.

L'usage de plateformes de curation de contenu me sert à enseigner aux étudiants comment choisir des catégories ou «tags » pour classer les informations utiles dans une mémoire à long terme, afin de les retrouver facilement par la suite. Cette compétence leur sera fort utile dans le reste de leur carrière.

Les blogs sont utilisés comme supports de « devoir final » pour les cours gradués (c'està-dire avant le master), et comme carnets de recherche pour les étudiants en maîtrise ou en doctorat : notes sur les lectures, formulation d'hypothèses, accumulation de données, première version d'articles scientifiques ou de chapitres des mémoires ou thèses, etc. Le carnet de recherche public facilite la relation avec le superviseur et permet de réorienter à temps les directions de recherche hasardeuses, d'entrer en contact avec les équipes travaillant sur les mêmes sujets, etc.

Le groupe Facebook est utilisé pour partager le Syllabus ou «plan de cours », l'agenda de la classe, les lectures obligatoires, les discussions internes au groupe - par exemple celles qui concernent l'évaluation - ainsi que les adresses électroniques des étudiants (Twitter, blog, plateforme de curation sociale, etc.). Toutes ces informations sont en ligne et accessibles d'un seul clic, y compris les lectures obligatoires. Les étudiants peuvent participer à l'écriture de mini-wikis à l'intérieur du groupe Facebook sur des sujets de leur choix, ils sont invités à suggérer des lectures intéressantes reliées au sujet du cours en ajoutant des liens commentés. J'utilise Facebook parce que la quasi-totalité des étudiants y sont déjà abonnés et que la fonctionnalité de groupe de cette plateforme 
est bien rodée. Mais j'aurais pu utiliser n'importe quel autre support de gestion de groupe collaboratif, comme Slack ou les groupes de Linkedin.

Sur Twitter, la conversation propre à chaque classe est identifiée par un hashtag. Au début, j'utilisais le médium à l'oiseau bleu de manière ponctuelle. Par exemple, à la fin de chaque classe je demandais aux étudiants de noter l'idée la plus intéressante qu'ils avaient retenu du cours et je faisais défiler leurs tweets en temps réel sur l'écran de la classe. Puis, au bout de quelques semaines, je les invitais à relire leurs traces collectives sur Twitter pour rassembler et résumer ce qu'ils avaient appris et poser des questions toujours sur Twitter - si quelque chose n'était pas clair, questions auxquelles je répondais par le même canal.

Au bout de quelques années d'utilisation de Twitter en classe, je me suis enhardi et je demande maintenant aux étudiants de prendre directement leurs notes sur ce medium social pendant le cours de manière à obtenir un cahier de notes collectif. Pouvoir regarder comment les autres prennent des notes (que ce soit sur le cours ou sur des textes à lire) permet aux étudiants de comparer leurs compréhensions et de préciser ainsi certaines notions. Ils découvrent ce que les autres ont relevé et qui qui n'est pas forcément ce qui les a stimulés eux-mêmes... Quand je sens que l'attention se relâche un peu, je leur demande de s'arrêter, de réfléchir à ce qu'ils viennent d'entendre et de noter leurs idées ou leurs questions, même si leurs remarques ne sont pas directement reliées au sujet du cours. Twitter leur permet de dialoguer librement entre eux sur les sujets étudiés sans déranger le fonctionnement de la classe. Je consacre toujours la fin du cours à une période de questions et de réponses qui s'appuie sur un visionnement collectif du fil Twitter. Cette méthode est particulièrement pertinente dans les groupes trop grands (parfois plus de deux cents personnes) pour permettre à tous les étudiants de s'exprimer oralement. Je peux répondre tranquillement aux questions après la classe en sachant que mes explications restent inscrites dans le fil du groupe. La conversation pédagogique se poursuit entre les cours.

En utilisant Facebook et Twitter en classe, les étudiants n'apprennent pas seulement la matière du cours mais aussi une façon «cultivée » de se servir des médias sociaux. Documenter ses petits déjeuners ou la dernière fête bien arrosée, disséminer des vidéos de chats et des images comiques, échanger des insultes entre ennemis politiques, s'extasier sur des vedettes du show-business ou faire de la publicité pour telle ou telle entreprise sont certainement des usages légitimes des médias sociaux. Mais on peut également entretenir des dialogues constructifs dans l'étude d'un sujet commun. On peut du même coup tisser des réseaux personnels d'apprentissage, c'est-à-dire collectionner des sources (individus, organisations) pertinentes pour se maintenir au courant dans les domaines d'expertise que l'on veut approfondir. (Dans le cas de Twitter, les réseaux personnels d'apprentissage prennent la forme de «listes» de 
personnes que l'on suit sur un sujet donné. Une liste bien construite permet de filtrer les tweets par sujets...) L'usage des médias sociaux en classe me permet de faire prendre conscience à mes étudiants qu'ils s'expriment dans une mémoire publique et qu'ils sont responsables des traces qu'ils y laissent, des idées qu'ils y diffusent, des émotions qu'ils y propagent. En somme, si petit soit-il, un Tweet ou un post sur Facebook sont déjà des «publications »: le télégramme social exprime publiquement un point de vue, cite d'autres usagers et renvoie à des données au moyen d'hyperliens. La responsabilité de son auteur s'étend évidemment aux gens qui le suivent et le lisent directement; mais elle comprend aussi les employeurs ou partenaires potentiels qui font des recherches sur sa personne; elle se prolonge enfin à toutes les recommandations et décisions politiques, économiques ou autres - qui suivent des traitements automatiques effectuées sur les messages en ligne! L'usage pédagogique de Facebook et Twitter semble paradoxal à de nombreux étudiants qui conçoivent les médias sociaux comme un espace « réservé aux adolescents », libre des contraintes imposées par les parents et l'école. Mais si cette conception des médias sociaux était encore valable au début des années 2000 elle ne l'est plus aujourd'hui puisque les médias sociaux sont devenus le moyen de communication dominant.

Quelles que soient les institutions dans lesquelles ils travaillent, j'estime que les enseignants devraient construire avec leurs étudiants des communautés ouvertes de pratique, de dialogue et de réflexion utilisant les plateformes gratuites qui sont déjà utilisées par les élèves et le grand public. Les plateformes fermées élèvent des murs virtuels qui bloquent les contacts transversaux pertinents avec des experts et d'autres communautés d'apprentissage. En revanche, l'usage de plateformes ouvertes inscrit résolument l'Université - et plus généralement l'école - dans le nouvel espace public. J'avoue que je ne crois pas aux « technologies éducatives ». Je donne ma préférence aux usages pédagogiques des techniques de communication grand public qui sont déjà utilisées par les étudiants. L'important n'est ni la plateforme, ni le logiciel ni la collection de ressources, mais les compétences cognitives trans-plateformes et transcontenus dont les pratiques éducatives doivent stimuler l'acquisition. Ce n'est pas Twitter qui doit polariser l'attention des étudiants, mais l'apprentissage de la clarté, de la brièveté et de la synthèse dans un dialogue où se construit la connaissance réflexive. De même, telle ou telle plateforme de curation de contenu n'est qu'un outil grâce auquel l'étudiant va s'initier à la catégorisation intelligente des données pour leur mutualisation dans une mémoire commune.

L'usage éducatif des média sociaux publics peut aller jusqu'à inclure les examens et les devoirs. J'ai expérimenté un « examen Twitter» où les étudiants devaient évaluer vingt de mes tweets en temps réel. Le code de communication était le suivant : pas de réaction 
s'ils pensaient que mon Tweet était faux, un coeur s'il contenait une part de vérité, un retweet s'ils étaient en gros d'accord et un retweet plus un coeur s'ils étaient complètement d'accord. Cela revenait à leur demander d'évaluer mes tweets sur une échelle de pertinence de 1 à 4. Après avoir relu avec eux mes tweets et les réponses qu'ils leur avaient donné, je leur demandais quel était, selon eux, la plus catastrophique des erreurs d'appréciation. Evidemment, les étudiants différaient dans leur estimation de la pire réponse. Je retenais toutes celles qu'ils avaient mentionnées et je retirais un point à tous ceux qui avaient donné l'une des mauvaises réponses identifiées par le groupe. Ainsi mon évaluation appliquait aux étudiants les règles qu'ils avaient eux-mêmes déterminées.

J'utilise maintenant une autre méthode d'évaluation, qui suppose la prise de note et le dialogue continu sur Twitter. Deux fois par semestre, les étudiants doivent relire la mémoire collaborative de la classe et sélectionner les éléments (notes de cours, questions, réponses, diagrammes, photos...) qui leur paraissent les plus intéressants ou les plus pertinents afin de construire un récit commenté de leur propre apprentissage. Ils peuvent pour ce faire utiliser les Moments de Twitter ou encore Storify. Au moyen de citations plus ou moins commentées, chaque étudiant produit alors son histoire d'apprentissage - son interprétation originale du cours - selon ses intérêts et sa subjectivité propre. Toutes les histoires d'apprentissage sont publiées sur Twitter avec le hashtag du cours, ce qui permet d'observer non seulement l'accumulation de la mémoire collective mais également la multitude des réflexions personnelles sur cette mémoire, avec leurs complémentarités et leurs divergences.

Dans les deux cas - qu'il s'agisse de l'estimation de la pertinence de mes tweets ou du résumé personnel du cours que je demande aux élèves - l'exercice évalué demande aux participants de faire un retour réflexif sur leur apprentissage collectif. A la fin du semestre, les étudiants ont non seulement acquis une connaissance du sujet enseigné mais ils ont aussi amélioré leurs compétences en apprentissage collaboratif dans un environnement trans-plateforme et ils ont peu ou prou expérimenté un processus d'intelligence collective réflexive dans la nouvelle sphère publique. Dans leur immense majorité, les étudiants apprécient un dispositif d'apprentissage dans lequel ils sont plus actifs, s'ennuient moins et apprennent mieux. Ce type d'expérimentation et de perfectionnement pédagogique est aujourd'hui exploré un peu partout dans le monde.

En réfléchissant sur ma pratique d'enseignant depuis une dizaine d'années, je réalise qu'elle repose sur un modèle de l'apprentissage collaboratif à trois phases : 1) une pratique commune, 2) un dialogue sur cette pratique, 3) une réflexion collective émergeant du dialogue et qui vient enrichir la pratique en retour. Dans mon cas, la pratique commune est fort simple puisqu'il s'agit de la prise de notes. Comme cette 
pratique est enregistrée et partagée en temps réel, elle implique immédiatement une activité collaborative et pose les bases du dialogue et de la réflexion ultérieure. Le dialogue, ou plutôt le multilogue, a lieu sur un mode transversal entre tous les membres de la communauté d'apprentissage et non seulement entre le professeur et les étudiants. Aussi bien les élèves que l'enseignant peuvent poser des questions et y répondre. Qui a besoin de conseils ou d'encouragements ? Comment reformuler telle ou telle notion? Tel exemple est-il pertinent? De nouvelles ressources ou références peuvent-elles faciliter la compréhension des élèves ? Une simple image trouvée sur Internet par un étudiant fait parfois la différence. Enfin, la réflexion s'appuie sur une relecture de la mémoire enregistrée de la classe. A la fin du semestre, les étudiants sont suffisamment familiers avec le sujet du cours pour créer un ou plusieurs documents multimédias où sont mis en oeuvre les compétences, connaissances et réflexions personnelles qui ont émergé du dialogue et de l'expérience gagnées par la fréquentation de la classe. Dès aujourd'hui, les étudiants en informatique publient leurs travaux et leurs discussions sur Github afin d'obtenir la reconnaissance de leurs pairs et d'afficher leurs compétences auprès des employeurs potentiels. Pourquoi les étudiants en sciences humaines ne suivraient-ils pas leurs traces?

Supposons maintenant qu'au lieu de représenter les connaissances acquises au moyen d'un diplôme ou d'un crédit (la cote d'un cours et la note obtenue), on les représente par un enregistrement de l'ensemble des transactions pédagogiques publiques auxquelles a participé un étudiant : pratiques, dialogues, oeuvres témoignant des compétences et de la réflexion développées (Grech and Camilleri, 2017). ... Ce qui serait enregistré et authentifié ne serait plus un bref document statique et relativement opaque - comme aujourd'hui - mais une fenêtre sur l'apprentissage collaboratif vivant où le professeur et les étudiants se rendent mutuellement témoignage. La garantie des apprentissages individuels ne serait plus séparée du processus d'intelligence collective d'où les savoirs ont émergé et où ils ont pris sens. Indépendamment des mutations institutionnelles et culturelles qu'elle implique, une telle évolution de la reconnaissance des savoirs serait aujourd'hui techniquement possible. Les blockchains sont des registres informatisés qui contiennent l'historique de tous les échanges et transactions effectués entre ses utilisateurs depuis leur création. Ces bases de données sont sécurisées par un procédé cryptographique et partagées par leurs différents utilisateurs sans intermédiaire. D'abord utilisée en finance et en comptabilité, la technologie de la blockchain se répand aujourd'hui dans d'autres secteurs d'application. L'éducation et - plus généralement l'authentification des expériences professionnelles et des compétences pourrait être une de ses futures applications vedette. Ainsi, à la fin d'un semestre, la mémoire de l'apprentissage collaboratif d'une classe, avec la participation de chaque étudiant, serait enregistrée et authentifiée par une blockchain. Cette nouvelle reconnaissance des 
savoirs amènerait un gain de transparence pour les contribuables qui financent l'éducation et pour les employeurs ou les collaborateurs potentiels des étudiants. Elle fournirait en outre aux institutions d'enseignement des flots de données fort précieuses pour étudier les évolutions cognitives et la qualité des apprentissages de leurs publics. De telles données seraient beaucoup plus précises et complètes que celles qui sont recueillies aujourd'hui au moyen d'évaluations après-coup et de sondages forcément partiels. Contrairement aux données récoltées au moyen des grilles fermées que l'on emploie souvent dans les enquêtes, elles ne préjugeraient pas des questions qui pourraient leur être posées. Les chercheurs pourraient interroger ces données au moyen d'algorithmes aussi variés que leurs hypothèses.

\section{Quelques extraits des tweets de mes classes : https://twitter.com/plevy/moments Hashtags de mes classes : \#UOKM \#UONM \#UOAC \#UOIM \#UOTM17}

\section{Ressources en ligne et exemples de curation par l'auteur}

- Personal and collective knowledge management: https://www.scoop.it/t/communication-and-km

- The rise of the algorithmic medium: https://www.scoop.it/t/the-rise-of-thealgorithmic-medium

- Humanities and their algorithmic revolution: https://www.scoop.it///digitalhumanities-for-beginners

- Education and cultural change: https://www.scoop.it/t/cultural-change

Presentación del artículo: 20 de diciembre de 2017 Fecha de aprobación: 13 de enero de 2018 Fecha de publicación: 31 de marzo de 2018

Lévy, P. (2018). Comment j'utilise les médias sociaux dans mes cours à l'université. RED. Revista de Educación a Distancia, 57. Consultado el (dd/mm/aaaa) en http://www.um.es/ead/red/57/levy_fr.pdf

\section{Financement}

Cette recherche n'a reçu aucune subvention spécifique de la part d'organismes de financement du secteur public, commercial ou à but non lucratif.

\section{Financiación}

Esta investigación no ha recibido ninguna subvención específica de los organismos de financiación en los sectores públicos, comerciales o sin fines de lucro. 


\section{BIBLIOGRAPHIE}

Barton, S. (2013). Social capital framework in the adoption of e-learning. International Jl. on E-Learning, 12 (2), 115-137.

Biskupic, I., Lackovic, S., \& Kresimir, J. (2015). Successful and proactive e-learning environment fostered by teachers' motivation in technology use. Social and Behavioral Sciences, 174, 3656-3662.

Bozanta, A., \& Mardikyan, S. (2017). The effects of social media use on collaborative learning: A case of Turkey. Turkish Online Journal of Distance Education, 18(1), 96-110.

Chugh, R., 2016. Harnessing social media as a knowledge management tool. IGI Global. Hershey, Pennsylvanie.

Cooke, S. (2017). Social teaching: Student perspectives on the inclusion of social media in higher education. Education and Information Technologies, 22(1), 255-269.

Craig-Hare, J. et al. (2017). The Effect of Socioscientific Topics on Discourse within an Online Game Designed to Engage Middle School Students in Scientific Argumentation, Journal of Education in Science, Environment and Health . DOI: 10.21891/jeseh.325783

Danley, St., Dahan, T., \& Benson, K. (2017). Publishing as social capital: Amplifying community with digital tools. Journal of Scholarly Publishing, 48(2), 116-130.

Grech, A. and Camilleri, A. F. (2017). Blockchain in Education. Inamorato dos Santos, A.(ed.) EUR 28778 EN; doi:10.2760/60649

Lepage, N. L. (2017). Des classes qui collaborent et apprennent avec Twitter. In Ecole branchée. Retrieved from: https://ecolebranchee.com/2017/01/19/classescollaborent-apprennent-twitter/

Levy, P. (2004). Inteligencia colectiva. Trad. Organización Panamericana de la Salud. Washington, DC. Retrieved from: http://inteligenciacolectiva.bvsalud.org/public/documents/pdf/es/inteligenciaC olectiva.pdf

Levy, P. (2011). The Semantic Sphere. Computation, cognition and information economy. ISTE / Wiley, Paris and London. 
Megele, C. (2015). eABLE. Embedding social media in academic curriculum as a learning and assessment strategy to enhance students learning and e-professionalism. Innovations in Education and Teaching International, 52(4), 414-425.

Redecker, C. Punie, Y (2017). Digital competence framework for educators, Publications office of the European Union, DOI online: 10.2760/159770

Reinie, L. and Wellman, B. 2012. Networked: The New Social Operating System, MIT Press, Cambridge Mass.

Rowland, A. et al. (2017). Social media: How the next generation can practice argumentation, Educational Media International. DOI: $10.1080 / 09523987.2017 .1362818$

Université Laval (2017). Guide sur l'utilisation des appareils mobiles en classe. Retrieved from: http://www.capres.ca/2017/05/guide-lutilisation-appareils-mobilesclasse/

Wan, Y. (2014). Building student trust in online learning environments, Distance Education, 35 (3), 345-359. 